\title{
On End-faithful Spanning Trees in Infinite Graphs
}

\author{
Reinhard Diestel
}

\section{Introduction}

Let $G$ be an infinite connected graph. A ray (from $v$ ) in $G$ is a 1-way infinite path in $G$ (with initial vertex $v$ ). An infinite connected subgraph of a ray $R \subset G$ is called a tail of $R$. If $X \subset G$ is finite, the infinite component of $R \backslash X$ will be called the tail of $R$ in $G \backslash X$.

The following assertions are equivalent for rays $P, Q \subset G$ :

(i) There exists a ray $R \subset G$ which meets each of $P$ and $Q$ infinitely often.

(ii) For every finite $X \subset G$, the tails of $P$ and $Q$ in $G \backslash X$ lie in the same component of $G \backslash X$.

(iii) $G$ contains infinitely many disjoint paths connecting a vertex of $P$ with one of $Q$.

If two rays $P, Q \subset G$ satisfy (i)-(iii), we call them end-equivalent (or briefly equivalent) in $G$ and write $P \underset{G}{\sim} Q$. An end of $G$ is an equivalence class under $\underset{G}{\sim}$, and $\mathcal{E}(G)$ denotes the set of ends of $G$. For example, the 2-way infinite ladder has two ends, the infinite grid $\mathbb{Z} \times \mathbb{Z}$ and every infinite complete graph have one end, and the dyadic tree has $2^{\aleph_{0}}$ ends.

This paper is concerned with the relationship between the ends of a connected infinite graph $G$ and the ends of its spanning trees. If $T$ is a spanning tree of $G$ and $P, Q$ are end-equivalent rays in $T$, then clearly $P$ and $Q$ are also equivalent in $G$. We therefore have a natural map $\eta: \mathcal{E}(T) \rightarrow \mathcal{E}(G)$ mapping each end of $T$ to the end of $G$ containing it. In general, $\eta$ need be neither 1-1 nor onto. For example, the 2-way infinite ladder has a spanning tree with 4 ends (the tree consisting of its two sides together with one rung), and every infinite complete graph is spanned by a star, which has no ends at all. A spanning tree $T$ of $G$ for which $\eta$ is $1-1$ is said to respect the ends of $G$ or called end-respecting, and a spanning tree $T$ for which $\eta$ is onto is called end-complete. An end-respecting and end-complete spanning tree is end-faithful.

The concept of an end was introduced for graphs by Halin [5] in 1964. It has since inspired some profound work in infinite graph theory; see for example Halin [6], Polat $[10,11]$, Seifter [12], Watkins [14], or any of several articles in [4]. The problem which Halin originally addressed in [5] is this:

Problem. Does every infinite connected graph have an end-faithful spanning tree?

Very recently, Seymour and Thomas [13] have been able to construct graphs which are infinitely connected - and hence have precisely one end - but in which every spanning tree 
has uncountably many ends. (One of their examples is $\mathrm{T} K_{\aleph_{1}}$-free, a fact which will lend additional relevance to the main result of this paper; see Theorem 1.3 below.) Thus, the general answer to Halin's question is no; the problem remains to understand what makes a graph contain an end-faithful spanning tree, and how these graphs can be recognized.

For countable graphs, a construction of an end-faithful spanning tree was already given as the main result in Halin [5]:

Theorem 1.1. [5] Every countable connected graph has an end-faithful spanning tree.

In 1969, Jung [8] investigated end-faithful spanning trees of a particularly intuitive kind: he characterized the graphs $G$ containing a normal rooted spanning tree $T$, one for which every pair of adjacent vertices of $G$ is comparable in the induced partial order $\frac{5}{T}$ on $V(G)$ (see below for an exact definition of $\frac{\leq}{T}$ ). Jung's characterization implies the following sharpening of Theorem 1.1:

Theorem 1.2. [8] Every countable connected graph has a normal rooted spanning tree.

The purpose of this paper is to construct an end-faithful spanning tree for any graph, irrespective of its cardinality, that does not contain a subdivided infinite complete graph as a subgraph:

Theorem 1.3. If $G$ is a connected graph not containing a $\mathrm{T} K_{\aleph_{0}}$, then $G$ has an endfaithful spanning tree $T$.

Our construction of the tree $T$ employs a certain decomposition of $G$ into countable factors, which enables us to use the end-faithful spanning trees constructed in the proof of Theorem 1.1. It should be emphasized that the mere existence of the spanning tree $T$ can be shown with considerably less effort by using the stronger Theorem 1.2 instead of Theorem 1.1 (Halin [7]).

The decomposition results needed for our construction of the tree $T$ are presented in Section 2. Most of these results have fairly straightforward proofs, found in [2]. Our key decomposition theorem however, Theorem 2.2, is proved in a separate paper [3]. Section 3 contains the construction of $T$. In Sections 4 and $5, T$ is shown to be end-faithful.

The terminology used in this paper is mostly standard, see e.g. [1]. In addition, we shall use the following notations.

If $P$ is a path with vertices $x$ and $y$, then $P_{x, y}$ denotes the subpath of $P$ from $x$ to $y$. If $P=x_{1} \ldots x_{n}$, then $\stackrel{\circ}{P}$ is the interior $x_{2} \ldots x_{n-1}$ of $P$. For $X, Y \subset G$, we call a path $P \subset G$ an $X-Y$ path if its endvertices are in $X$ and $Y$, respectively, and its interior vertices are in $G \backslash(X \cup Y)$. 
If $T$ is a rooted tree, with root $v_{0}$ say, then $T$ induces a natural partial order $\underset{T}{\leq}$ on its vertices: $v \leq \frac{\leq}{T} w$ if $v$ lies on the unique $v_{0}-w$ path in $T$. A ray, for the purpose of this definition, will be assumed to be rooted at its initial vertex.

A graph $H \subset G$ is called convex in $G$ if $H$ contains every induced path in $G$ whose endvertices are in $H$. Equivalently, $H$ is convex in $G$ if and only if the endvertices of every $H-H$ path in $G$ are adjacent in $H$.

$G$ is locally finite if every vertex of $G$ has finite degree. By a well-known theorem of König [9], every infinite but locally finite connected graph contains a ray from each of its vertices.

And finally, if $G$ is a graph and $a$ is a cardinal, the $a$-closure of $G$ is obtained from $G$ by adding all edges $x y \notin E(G)$ for which $G$ contains $a$ independent $x-y$ paths (see [3]).

In our construction of the tree $T$ for Theorem 1.3, we shall use Theorem 1.1 in the following slightly sharper version:

Theorem 1.1'. If $G$ is a countable connected graph and $F \subset G$ is a finite forest, then $G$ has an end-faithful spanning tree $T$ which contains $F$.

Proof. Use Theorem 1.1 to find an end-faithful spanning tree in every component of $G \backslash F$. Extend the union of these trees with $F$ to a spanning tree $T$ of $G$. $T$ is end-faithful in $G$.

\section{Simplicial decompositions and tree-decompositions.}

Let $G$ be a graph, $\sigma>0$ an ordinal, and let $B_{\lambda}$ be an induced subgraph of $G$ for every $\lambda<\sigma$. The family $F=\left(B_{\lambda}\right)_{\lambda<\sigma}$ is called a simplicial decomposition of $G$ if the following three conditions hold:

(S1) $G=\bigcup_{\lambda<\sigma} B_{\lambda}$

(S2) $\left(\bigcup_{\lambda<\mu} B_{\lambda}\right) \cap B_{\mu}=: S_{\mu}$ is a complete graph for each $\mu$

$$
\begin{array}{r}
(0<\mu<\sigma) ; \\
(0 \leq \lambda<\mu<\sigma) .
\end{array}
$$

(S3) no $S_{\mu}$ contains $B_{\mu}$ or any other $B_{\lambda}$

For $v \in V(G)$ and $H \subset G$, we denote by $\lambda(v)$ the minimal $\lambda<\sigma$ for which $v \in B_{\lambda}$, and set $\Lambda(H):=\{\lambda(v) \mid v \in V(H)\}$. Then $\lambda(v)=\mu$ if and only if $v \in B_{\mu} \backslash S_{\mu}$, and $\lambda(v)<\mu$ for all $v \in S_{\mu}$. For $\mu \leq \sigma$, we write $\left.G\right|_{\mu}:=\bigcup_{\lambda<\mu} B_{\lambda}$.

We shall usually refer to a complete graph as a simplex, as is the custom in the field. The graphs $S_{\mu}=\left.G\right|_{\mu} \cap B_{\mu}$ in (S2) will be called simplices of attachment.

In a simplicial decomposition, each simplex of attachment $S_{\mu}$ is by definition contained in the union of the factors $B_{\lambda}, \lambda<\mu$. In many simplicial decompositions, including all those of finite graphs, each $S_{\mu}$ is even contained in just one of the earlier factors [2]: 
(S4) each $S_{\mu}$ is contained in $B_{\lambda}$ for some $\lambda<\mu$

When this happens, we denote by $\tau(\mu)$ the minimal $\lambda<\mu$ for which $S_{\mu} \subset B_{\lambda}$, and inductively define $\tau^{k}(\mu):=\tau\left(\tau^{k-1}(\mu)\right)$, where $\tau^{0}(\mu)=\mu$.

A family $F=\left(B_{\lambda}\right)_{\lambda<\sigma}$ which satisfies (S1) and (S4) (but not necessarily (S2) or (S3)) is called a tree-decomposition of $G$, and if $F$ satisfies all of (S1)-(S4), it is called a simplicial tree-decomposition of $G$. The reason for this is that we can associate with $F$ a decomposition tree $T_{F}=T_{F}(G)$, as follows:

$$
\begin{aligned}
& V\left(T_{F}\right):=\left\{B_{\lambda} \mid \lambda<\sigma\right\}, \\
& E\left(T_{F}\right):=\left\{B_{\mu} B_{\tau(\mu)} \mid \mu<\sigma\right\} .
\end{aligned}
$$

The first factor in $F, B_{0}$, is taken to be the root of $T_{F}$. Thus $B_{\mu} \underset{T_{F}}{\leq} B_{\nu}$ if and only if $\mu=\tau^{k}(\nu)$ for some $k \geq 0$. Furthermore, $\Lambda\left(S_{\mu}\right) \subset\left\{\tau^{k}(\mu) \mid k \in \mathbb{N}\right\}$ (induction on $\mu$ ), so in particular $B_{\lambda(v)} \underset{T_{F}}{\leq} B_{\mu}$ for all $v \in B_{\mu}$ (see [2] for details). If a graph $G$ contains no infinite simplex, its rays are closely related to the rays in its decomposition tree. This fact will be central to our construction of the tree $T$.

The decompositions we shall use will have another property: they are coherent. A decomposition $\left(B_{\lambda}\right)_{\lambda<\sigma}$ is coherent if, for every $\lambda<\sigma$, each vertex of $S_{\lambda}$ has a neighbour in $B_{\lambda} \backslash S_{\lambda}$, and $B_{\lambda} \backslash S_{\lambda}$ is connected.

We now list a number of facts about simplicial decompositions and tree-decompositions that will be used later. The first of these facts is a fundamental property of the factors in a simplicial decomposition.

Proposition 2.1. [2] If $\left(B_{\lambda}\right)_{\lambda<\sigma}$ is a simplicial decomposition of $G$, then every $B_{\mu}$ is a convex subgraph of $G$.

The next theorem will be our main tool. Its proof is given in [3].

Theorem 2.2. Let $G$ be an uncountable graph not containing any subdivided infinite simplex. Then the $\aleph_{1}$-closure $G^{\prime}$ of $G$ admits a coherent simplicial tree-decomposition $F=\left(B_{\lambda}\right)_{\lambda<\sigma}$ into countable factors and with finite simplices of attachment, which has the following property: for every $\mu<\sigma$ and every edge $x y \in\left(E\left(B_{\mu}\right) \backslash E\left(S_{\mu}\right)\right) \backslash E(G)$, there are uncountably many ordinals $\nu$, with $\tau(\nu)=\mu$, such that $B_{\nu}$ contains an $S_{\nu}-S_{\nu}$ path $P$ with endvertices $x, y$ and $E(P) \subset E(G)$.

The remaining two propositions concern separation properties in tree-decompositions. Their proofs are straightforward throughout. [2]

Proposition 2.3. If $B, B^{\prime}, B^{\prime \prime}$ are factors in a tree-decomposition $F$ of $G$ and $B$ lies on the $B^{\prime}-B^{\prime \prime}$ path in $T_{F}(G)$, then $B$ separates $B^{\prime} \backslash B$ from $B^{\prime \prime} \backslash B$ in $G$. 
Proposition 2.4. Let $F=\left(B_{\lambda}\right)_{\lambda<\sigma}$ be a tree-decomposition of $G$. Let $\lambda, \mu, \nu<\sigma$ be such that $B_{\nu} \underset{T_{F}}{\geq} B_{\mu}$ but $B_{\lambda} \underset{T_{F}}{\gtrless} B_{\mu}$. Then $S_{\mu}$ separates $B_{\lambda} \backslash S_{\mu}$ from $B_{\nu} \backslash S_{\mu}$ in $G$.

\section{The construction of $T$}

Let $G$ be a given uncountable graph, $G \not \supset \mathrm{T} K_{\aleph_{0}}$. We shall construct an end-faithful spanning tree $T$ of $G$.

The basic idea for the construction of $T$ is as follows. Using Theorem 2.2, we decompose the $\aleph_{1}$-closure $G^{\prime}$ of $G$ into countable factors $B_{\mu}, \mu<\sigma$. By Halin's theorem, we can then find an end-faithful spanning tree $T_{\mu}$ in each of the factors $B_{\mu}$. Essentially, our task will be to choose the trees $T_{\mu}$ in such a way that they can be pieced together inductively to form $T$, our desired end-faithful spanning tree of $G$.

There are various problems we have to be aware of during the construction of $T$. One of them lies in the fact that the trees $T_{\mu}$ will in general contain edges from $E\left(G^{\prime}\right) \backslash E(G)$, which must be replaced by paths in $G$ before $T_{\mu}$ can be incorporated into $T$. In replacing these edges, we have to ensure that their replacement paths are pairwise independent and avoid the part of $T$ already constructed. Conversely, we shall not be entirely free in choosing $T_{\mu}$, because replacement paths corresponding to earlier trees $T_{\lambda}(\lambda<\mu)$ may have spilled over into $B_{\mu}$ and have to be accommodated into $T_{\mu}$. This problem will be taken care of by Theorem 2.2, which was tailored specificly for this purpose.

Another problem deserving attention is that of stringing the $T_{\mu}$ 's together in the right way, so that $T$ does indeed emerge as end-faithful when the construction is complete. To deal with this problem, we shall rely on the close relationship between the ends of $G$ and those of the decomposition tree $T_{F}\left(G^{\prime}\right)$ belonging to our simplicial tree-decomposition $F$ of $G^{\prime}$. This relationship is based on the fact that all the simplices of attachment in $F$ are finite: since a ray in $G$ can pass only finitely often through any given $S_{\mu}$, it must either be 'centred on' (have infinitely many vertices in) one factor $B_{\mu}$, or follow the course of a ray in $T_{F}$. Moreover, equivalent rays in $G$ must follow the same ray in $T_{F}$, because their tails cannot be separated by a finite set of vertices (cf. Proposition 2.4). In this way, each end of $G$ induces an end of $T_{F}$ (or collapses to one vertex $B_{\mu}$ of $T_{F}$ ).

Since equivalent rays in $G$ follow a unique ray in $T_{F}$ (ore none at all), $T$ can only be end-faithful if it contains, for each end $E$ of $T_{F}$, a unique ray $Q$ (from a fixed root $v_{0}$ ) that induces $E$. The uniqueness of these rays $Q \subset T$ will be ensured by specifying a single vertex $s_{\mu}$ in every $S_{\mu}$, to serve as a bottle-neck for all paths in $T$ passing from $\left.G^{\prime}\right|_{\mu}$ into $B_{\mu} \backslash S_{\mu}$. The existence of the rays $Q$ will be guaranteed by the specific choice of $s_{\mu}$ in $S_{\mu}$. Figure 1 shows examples of how failure to select bottle-neck vertices $s_{\mu} \in S_{\mu}$ at all or a wrong choice of $s_{\mu}$ 's may result in a spanning tree $T$ that fails to be end-respecting or end-complete, respectively. 

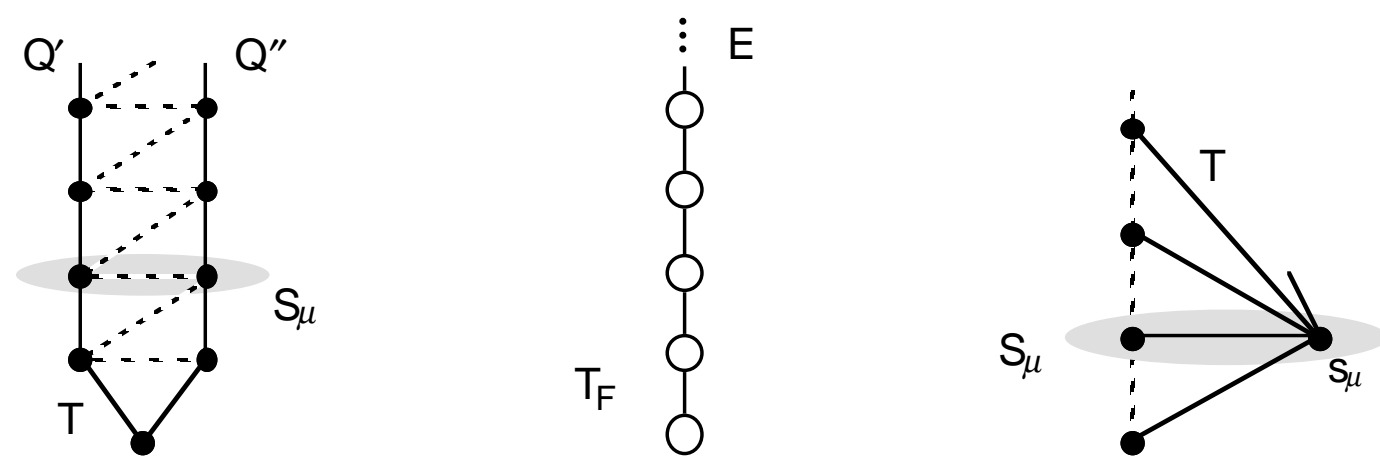

FIGURE 1. End-unfaithful spanning trees in graphs with simplicial decompositions into triangles

Before we begin our formal construction of $T$, let us run through some of the terms that will be used. $F=\left(B_{\lambda}\right)_{\lambda<\sigma}$ will be a fixed simplicial tree-decomposition of the $\aleph_{1^{-}}$ closure $G^{\prime}$ of $G$ into countable factors $B_{\lambda}$. The tree $T$ will be obtained as the union of a nested sequence of graphs $\left.T\right|_{\mu}, \mu<\sigma$. Each $\left.T\right|_{\mu}$ will have the following properties:

$\mathbf{A}_{1}(\mu):\left.\quad T\right|_{\mu} \subset G,\left.T\right|_{\mu}$ is a tree, and $V\left(\left.T\right|_{\mu}\right) \supset V\left(\left.G^{\prime}\right|_{\mu}\right)$. Moreover, $\left.\left.T\right|_{\mu} \supset T\right|_{\lambda}$ for every $\lambda<\mu$.

For each $\mu<\sigma$, the definition of $\left.T\right|_{\mu+1}$ will depend on $\left.T\right|_{\mu}$, on another graph $T_{\mu}$, and on the choice of a certain edge $e_{\mu}$. Here,

$\mathbf{A}_{2}(\mu): \quad T_{\mu}$ is an end-faithful spanning tree of $B_{\mu} \backslash S_{\mu} ; T_{\mu}$ may have edges that are not edges of $G$.

$\mathbf{A}_{3}(\mu): \quad e_{\mu}$ is an edge of $G^{\prime}$ joining $T_{\mu}$ to $\left.T\right|_{\mu} \cap S_{\mu}$ in such a way that $\left(\left.T_{\mu} \cup T\right|_{\mu}\right)+e_{\mu}$ is a tree.

The term $E_{\mu}$ will denote the set of edges $e \in E\left(T_{\mu}\right) \cup\left\{e_{\mu}\right\}$ that are not edges of $G$. When $\left.T\right|_{\mu+1}$ is formed from the union of $\left.T\right|_{\mu}, T_{\mu}$ and $\left\{e_{\mu}\right\}$, these edges $e$ are replaced with independent paths $P(e) \subset G$. By Theorem 2.2, these paths will be chosen in such a way that they run through different factors $B_{\nu(e)}, \nu(e)>\mu$, for different edges $e \in E_{\mu}$. This scattering of the paths $P(e)$ will have the desired effect that any spillover of $\left.T\right|_{\mu}$ into $B_{\nu} \backslash S_{\nu}$ (for some fixed $\nu \geq \mu$ ) remains unchanged as $\mu$ grows towards $\nu$, and therefore keeps its original form of $P(e)$ :

$\mathbf{A}_{4}(\mu):$ If $\mu \leq \nu<\sigma$ and $\left.T\right|_{\mu} \cap B_{\nu} \not \subset S_{\nu}$, then $\left.T\right|_{\mu} \cap B_{\nu}=\left(\left.T\right|_{\mu} \cap S_{\nu}\right) \cup P$, where $P$ is an $S_{\nu}-S_{\nu}$ path in $B_{\nu}$. Moreover, there exist $\lambda<\mu$ and an edge $e \in E_{\lambda}$, such that $\lambda=\tau(\nu), \quad \nu=\nu(e)$ and $P=P(e)$.

We are now ready to begin the formal construction of $T$, an end-faithful spanning tree of our graph $G$.

Let $G^{\prime}$ be the $\aleph_{1}$-closure of $G$, and let $F=\left(B_{\lambda}\right)_{\lambda<\sigma}$ be a simplicial tree-decomposition of $G^{\prime}$ as provided by Theorem 2.2. Let $v_{0}$ be a vertex of $B_{0} ; v_{0}$ will be kept fixed throughout the proof and serve as the root of $T$. Since $F$ is a tree-decomposition, it has a 
decomposition tree $T_{F}$, whose vertices are the factors $B_{\lambda}, \lambda<\sigma$. For simplicity, we shall normally use $\leq$ rather than $\underset{T_{F}}{\leq}$ to denote the natural partial order on $V\left(T_{F}\right)$.

Since any two vertices $x, y \in G^{\prime}$ with $x y \in E\left(G^{\prime}\right) \backslash E(G)$ are joined in $G$ by uncountably many independent paths (recall the definition of the $\aleph_{1}$-closure), the replacement of such edges with suitable paths in $G$ yields the following:

Lemma 3.1. If $P, Q \subset G$ are rays with $P \underset{G^{\prime}}{\sim} Q$, then $P \underset{G}{\sim} Q$.

Let $0 \leq \mu \leq \sigma$, and suppose that for all $\lambda<\mu$ we have defined $\left.T\right|_{\lambda},\left.T\right|_{\lambda+1}, T_{\lambda}, e_{\lambda}$, $s_{\lambda}$ and $E_{\lambda}$, together with $\nu(e)$ and $P(e)$ for all $e \in E_{\lambda}$, such that $\mathbf{A}_{1}(\lambda), \mathbf{A}_{1}(\lambda+1), \mathbf{A}_{4}(\lambda)$ and $\mathbf{A}_{4}(\lambda+1)$ hold. In the following we shall define the above terms for $\lambda=\mu$ and prove $\mathbf{A}_{1}$ and $\mathbf{A}_{4}$ for $\mu$ and $\mu+1$, provided that $\mu, \mu+1<\sigma$ (except that for the definition of $\left.T\right|_{\mu}$ and the proofs of $\mathbf{A}_{1}(\mu)$ and $\mathbf{A}_{4}(\mu)$ we include the case of $\mu=\sigma$, and that $e_{\mu}$ will only be defined for $\mu>0$ ).

We first define $\left.T\right|_{\mu}$ and prove $\mathbf{A}_{1}(\mu)$ and $\mathbf{A}_{4}(\mu)$. If $\mu$ is a successor ordinal, $\mu=\lambda+1$ say, then $\left.T\right|_{\mu}$ is already defined, and $\mathbf{A}_{1}(\mu)$ and $\mathbf{A}_{4}(\mu)$ hold by assumption. Suppose therefore that $\mu$ is not a successor ordinal. If $\mu=0$, set $\left.T\right|_{\mu}:=\emptyset$; then $\mathbf{A}_{1}(\mu)$ and $\mathbf{A}_{4}(\mu)$ hold trivially. Otherwise, i.e. if $\mu$ is a limit ordinal, let

$$
\left.T\right|_{\mu}:=\left.\bigcup_{\lambda<\mu} T\right|_{\lambda}
$$

$\mathbf{A}_{1}(\mu)$ is easily seen to follow from our assumption that $\mathbf{A}_{1}(\lambda)$ holds for all $\lambda<\mu$. To verify $\mathbf{A}_{4}(\mu)$, notice that if $\mu \leq \nu<\sigma$ and $\left.T\right|_{\mu} \cap B_{\nu} \not \subset S_{\nu}$, then also $\left.T\right|_{\lambda^{\prime}} \cap B_{\nu} \not \subset S_{\nu}$ for some $\lambda^{\prime}<\mu$, since $\mu$ is a limit ordinal. By $\mathbf{A}_{4}\left(\lambda^{\prime}\right)$,

$$
\left.T\right|_{\lambda^{\prime}} \cap B_{\nu}=\left(\left.T\right|_{\lambda^{\prime}} \cap S_{\nu}\right) \cup P
$$

where $P=P(e)$ for some $e \in E_{\lambda}$ with $\lambda<\lambda^{\prime}, \quad \lambda=\tau(\nu)$ and $\nu=\nu(e)$. Let $\lambda^{\prime \prime}$ be any ordinal with $\lambda^{\prime} \leq \lambda^{\prime \prime}<\mu$. Clearly again $\left.T\right|_{\lambda^{\prime \prime}} \cap B_{\nu} \not \subset S_{\nu}$, because $\left.\left.T\right|_{\lambda^{\prime \prime}} \supset T\right|_{\lambda^{\prime}}$. Thus, by $\mathbf{A}_{4}\left(\lambda^{\prime \prime}\right),\left.\quad T\right|_{\lambda^{\prime \prime}} \cap B_{\nu}$ has the form $\left(\left.T\right|_{\lambda^{\prime \prime}} \cap S_{\nu}\right) \cup \widetilde{P}$, where $\widetilde{P}$ is an $S_{\nu}-S_{\nu}$ path in $B_{\nu}$. But $\widetilde{P} \supset P$ (again by $\left.\left.T\right|_{\lambda^{\prime \prime}} \supset T\right|_{\lambda^{\prime}}$ ), and $P$ is also an $S_{\nu}-S_{\nu}$ path. Therefore $\widetilde{P}=P=P(e)$. Thus

$$
\begin{aligned}
\left.T\right|_{\mu} \cap B_{\nu} & =\bigcup_{\lambda^{\prime} \leq \lambda^{\prime \prime \prime}<\mu}\left(\left.T\right|_{\lambda^{\prime \prime}} \cap B_{\nu}\right) \\
& =\bigcup_{\lambda^{\prime} \leq \lambda^{\prime \prime \prime}<\mu}\left(\left.T\right|_{\lambda^{\prime \prime}} \cap S_{\nu}\right) \cup P(e) \\
& =\left(\left.T\right|_{\mu} \cap S_{\nu}\right) \cup P(e),
\end{aligned}
$$

where $P(e)$ is an $S_{\nu}-S_{\nu}$ path in $B_{\nu}$ and $e \in E_{\lambda}$ with $\lambda<\lambda^{\prime}<\mu, \lambda=\tau(\nu)$ and $\nu=\nu(e)$. This completes the proof of $\mathbf{A}_{4}(\mu)$. 
For the remaining definitions and the proofs of $\mathbf{A}_{1}(\mu+1)$ and $\mathbf{A}_{4}(\mu+1)$, we shall assume that $\mu \neq \sigma$. Let us say that $\mu$ is of type 1 if $\left.T\right|_{\mu} \cap B_{\mu} \subset S_{\mu}$, and of type 2 otherwise.

For the definition of $T_{\mu}$, recall that since $F$ is coherent, $B_{\mu} \backslash S_{\mu}$ is connected. If $\mu$ is of type 1, then $\left.T\right|_{\mu} \cap\left(B_{\mu} \backslash S_{\mu}\right)=\emptyset$, and we let $T_{\mu}$ be any end-faithful spanning tree of $B_{\mu} \backslash S_{\mu}$. If $\mu$ is of type 2, then $\left.T\right|_{\mu} \cap\left(B_{\mu} \backslash S_{\mu}\right)=\stackrel{\circ}{P}(e)$ for some $e \in E_{\lambda}$ and $\lambda<\mu$ (by $\mathbf{A}_{4}(\mu)$ ), and we let $T_{\mu}$ be an end-faithful spanning tree of $B_{\mu} \backslash S_{\mu}$ containing $\stackrel{\circ}{P}(e)$. These choices of $T_{\mu}$ are possible by Theorems 1.1 and $1.1^{\prime}$, and they satisfy $\mathbf{A}_{2}(\mu)$.

Next we define $e_{\mu}$ and $s_{\mu}$. Set $s_{0}:=v_{0}$, and assume in the sequel that $\mu>0$. If $\mu$ is of type 1 , we let $e_{\mu}$ be any edge $x y$ of $G^{\prime}$ with $\lambda(x)=\tau(\mu)$ and $\lambda(y)=\mu$. Notice that this choice of $e_{\mu}$ is always possible: by definition of $\tau(\mu), S_{\mu}$ has a vertex $x$ with $\lambda(x)=\tau(\mu)$, and $x$ has a neighbour $y$ in $B_{\mu} \backslash S_{\mu}$, because $F$ is coherent. If $\mu$ is of type 2 on the other hand, we let $e_{\mu}$ be the unique edge that lies on the $v-v_{0}$ path in $\left.T\right|_{\mu}$ for every $v \in \stackrel{\circ}{P}(e)$ ( $e \in E_{\lambda}$ as earlier). The existence and uniqueness of such an edge follow from the fact that $\left.T\right|_{\mu}$ is a tree $\left(\mathbf{A}_{1}(\mu)\right)$ and that every $v \in \stackrel{\circ}{P}(e)$ has degree 2 in $\left.T\right|_{\mu}\left(\mathbf{A}_{4}(\mu)\right)$. In each case, $\left(\left.T_{\mu} \cup T\right|_{\mu}\right)+e_{\mu}$ is a tree $\left(\mathbf{A}_{3}(\mu)\right)$.

Notice also that in both cases the definition of $e_{\mu}$ is such that $e_{\mu}$ has one endvertex in $S_{\mu}$ and one in $B_{\mu} \backslash S_{\mu}$; we let $s_{\mu}$ be the endvertex of $e_{\mu}$ in $S_{\mu}$. Then

$\mathbf{B}(\mu)$ : For every $v \in B_{\mu} \backslash S_{\mu}$, the vertex $s_{\mu}$ lies on the $v-v_{0}$ path in the tree $\left(\left.T_{\mu} \cup T\right|_{\mu}\right)+e_{\mu}$.

Let

$$
E_{\mu}:=\left(E\left(T_{\mu}\right) \cup\left\{e_{\mu}\right\}\right) \backslash E(G) .
$$

Using the property of $F$ given by Theorem 2.2, we now choose for each edge $x y \in E_{\mu}$ an ordinal $\nu=: \nu(e)$ with $\tau(\nu)=\mu$, such that $B_{\nu}$ contains an $S_{\nu}-S_{\nu}$ path $P$ with endvertices $x, y$ and $E(P) \subset E(G)$; the path $P$ will be denoted by $P(e)$. Moreover, we choose the ordinals $\nu(e)$ in such a way that $\nu(e) \neq \nu\left(e^{\prime}\right)$ for distinct $e, e^{\prime} \in E_{\mu}$; this is again possible by Theorem 2.2, because $\left|E_{\mu}\right| \leq\left|B_{\mu}\right|^{2} \leq \aleph_{0}$. Since $\lambda(v)=\nu(e)>\mu$ for every $v \in \stackrel{\circ}{P}(e)$ with $e \in E_{\mu}$, the following holds:

$\mathbf{C}(\mu): \quad$ If $e, e^{\prime} \in E_{\mu}$ and $e \neq e^{\prime}$, then $\stackrel{\circ}{P}(e) \cap \stackrel{\circ}{P}\left(e^{\prime}\right)=\emptyset$ and $\left.\stackrel{\circ}{P}(e) \cap G^{\prime}\right|_{\mu+1}=\emptyset$.

It remains to define $\left.T\right|_{\mu+1}$ and to prove $\mathbf{A}_{1}(\mu+1)$ and $\mathbf{A}_{4}(\mu+1)$. Let us set

$$
\left.T\right|_{\mu+1}:=\left(\left(\left(\left.T_{\mu} \cup T\right|_{\mu}\right)+e_{\mu}\right) \cup \bigcup_{e \in E_{\mu}} P(e)\right)-E_{\mu} .
$$

In order to prove $\mathbf{A}_{1}$ and $\mathbf{A}_{4}$ for $\mu+1$, observe first that the sets

$$
N_{\lambda}:=\left\{\nu(e) \mid e \in E_{\lambda}\right\}
$$

are disjoint for distinct values of $\lambda \leq \mu$, because $\tau(\nu)=\lambda$ for all $\nu \in N_{\lambda}$ (by definition of $\nu(e))$. In particular,

$$
e \in E_{\lambda}, e^{\prime} \in E_{\lambda^{\prime}}, \lambda \neq \lambda^{\prime} \Rightarrow \stackrel{\circ}{P}(e) \cap \stackrel{\circ}{P}\left(e^{\prime}\right)=\emptyset .
$$


By $\mathbf{A}_{4}(\mu)$, any vertex $v$ of $\left.\left.T\right|_{\mu} \backslash G^{\prime}\right|_{\mu+1}$ must be on some $P(e)$ with $e \in E_{\lambda}, \quad \lambda<\mu$. Hence (1) implies that $\left(\left.\left.T\right|_{\mu} \backslash G^{\prime}\right|_{\mu+1}\right) \cap \stackrel{\circ}{P}(e)=\emptyset$ for all $e \in E_{\mu}$. Combining this with $\mathbf{C}(\mu)$, we obtain

$\mathbf{D}(\mu):\left.\quad T\right|_{\mu} \cap \stackrel{\circ}{P}(e)=\emptyset$, for all $e \in E_{\mu}$.

As $\left(\left.T_{\mu} \cup T\right|_{\mu}\right)+e_{\mu}$ is a tree, and the paths $\stackrel{\circ}{P}(e), e \in E_{\mu}$, are pairwise disjoint and avoid $T_{\mu}$, $\mathbf{D}(\mu)$ implies that $\left.T\right|_{\mu+1}$ is a tree. This establishes $\mathbf{A}_{1}(\mu+1)$, the other assertions being obvious.

For the proof of $\mathbf{A}_{4}(\mu+1)$, let $\nu$ with $\mu+1 \leq \nu<\sigma$ and $\left.T\right|_{\mu+1} \cap B_{\nu} \not \subset S_{\nu}$ be given. If $\nu \in N_{\mu}$, say $\nu=\nu(e)$ with $e \in E_{\mu}$, then $\nu \notin N_{\lambda}$ for all $\lambda<\mu$, and hence $\left.T\right|_{\mu} \cap B_{\nu} \subset S_{\nu}$ by $\mathbf{A}_{4}(\mu)$. Thus $\left.T\right|_{\mu} \cap\left(B_{\nu} \backslash S_{\nu}\right)=\emptyset$, and therefore $\left.T\right|_{\mu+1} \cap\left(B_{\nu} \backslash S_{\nu}\right)=\stackrel{\circ}{P}(e)$. This implies

$$
\left.T\right|_{\mu+1} \cap B_{\nu}=\left(\left.T\right|_{\mu+1} \cap S_{\nu}\right) \cup P(e),
$$

as desired. On the other hand if $\nu \notin N_{\mu}$, then $\left.\left.T\right|_{\mu+1} \cap\left(B_{\nu} \backslash S_{\nu}\right) \subset T\right|_{\mu}$, so

$$
\left.T\right|_{\mu+1} \cap\left(B_{\nu} \backslash S_{\nu}\right)=\left.T\right|_{\mu} \cap\left(B_{\nu} \backslash S_{\nu}\right)=\stackrel{\circ}{P}(e)
$$

for some $e \in E_{\lambda}$ and $\lambda<\mu$, again by $\mathbf{A}_{4}(\mu)$. Thus again

$$
\left.T\right|_{\mu+1} \cap B_{\nu}=\left(\left.T\right|_{\mu+1} \cap S_{\nu}\right) \cup P(e),
$$

completing the proof of $\mathbf{A}_{4}(\mu+1)$.

Let us finally set

$$
T:=\left.T\right|_{\sigma} .
$$

By $\mathbf{A}_{1}(\sigma), T$ is a spanning tree of $G$.

The proof that $T$ is end-faithful with respect to $G$ will be given in Sections 4 and 5 . In the remainder of this section we shall extract a few facts from the construction of $T$ for later use. Unless otherwise stated, each of these facts holds for every $\mu<\sigma$.

The first fact concerns the edges in the sets $E_{\mu}$.

$\mathbf{E}(\mu): \quad$ If $e=x y \in E_{\mu}$ and $\lambda(x) \leq \lambda(y)$, then either $\lambda(x)=\lambda(y)=\mu$, or $\lambda(x)=\tau(\mu)$, $\lambda(y)=\mu$ and $x=s_{\mu}$.

For the proof of $\mathbf{E}(\mu)$, notice first that if $e \in E\left(T_{\mu}\right)$, then $\lambda(x)=\lambda(y)=\mu$ by $T_{\mu} \subset B_{\mu} \backslash S_{\mu}$. Suppose therefore that $e \notin E\left(T_{\mu}\right)$, i.e. that $e=e_{\mu}$. Then $\mu$ must be of type 1 , since otherwise $e_{\mu}$ would be in $E\left(\left.T\right|_{\mu}\right) \subset E(G)$, and hence not in $E_{\mu}$. Therefore $\lambda(x)=\tau(\mu)$, $\lambda(y)=\mu$ and $x=s_{\mu}$ by definition of $e_{\mu}$ and $s_{\mu}$.

The second fact contains the information ensuring that the definition of $s_{\mu}$ achieves its purpose; see our earlier informal discussion.

$\mathbf{F}(\mu): \quad \lambda\left(s_{\mu}\right) \in\left\{\tau(\mu), \tau^{2}(\mu)\right\}$, and if $\lambda\left(s_{\mu}\right)=\tau^{2}(\mu)$, then $s_{\mu}=s_{\tau(\mu)}($ for $\mu>0)$.

The proof of $\mathbf{F}(\mu)$ is clear by definition of $e_{\mu}$ if $\mu$ is of type 1 (and hence $\lambda\left(s_{\mu}\right)=\tau(\mu)$ ). If $\mu$ is of type 2 , then $s_{\mu}$ is an endvertex of $P(e)$ and hence of $e$ for some $e \in E_{\lambda}$ with 
$\lambda=\tau(\mu)$. By $\mathbf{E}(\lambda)$, this implies that either $\lambda\left(s_{\mu}\right)=\lambda=\tau(\mu)$, or else $\lambda\left(s_{\mu}\right)=\tau(\lambda)=\tau^{2}(\mu)$ and $s_{\mu}=s_{\lambda}=s_{\tau(\mu)}$.

Let us note the following immediate consequence of $(\mathrm{F})$.

If $B_{\lambda_{0}} B_{\lambda_{1}} \ldots$ is a ray from $B_{0}$ in $T_{F}$, then $\left\{s_{\lambda_{i}} \mid i \in \mathbb{N}\right\}$ is infinite.

Indeed, as $B_{\lambda_{0}}=B_{0}$, we have $\tau\left(\lambda_{n}\right)=\lambda_{n-1}$ for all $n \in \mathbb{N}$. Therefore $\lambda\left(s_{\lambda_{n}}\right) \in\left\{\lambda_{n-1}, \lambda_{n-2}\right\}$ (by $\mathbf{F}\left(\lambda_{n}\right)$ ), for all $n \in \mathbb{N}$. This implies (2).

Next, we show that $T$ contains essentially no $B_{\mu}-B_{\mu}$ paths other than those of the form $P(e), e \in E_{\mu}$.

$\mathbf{G}(\mu)$ If $P$ is a $B_{\mu}-B_{\mu}$ path in $T$, with endvertices $x$ and $y$ where $y \in B_{\mu} \backslash S_{\mu}$, then $x y \in E_{\mu}$ and $P=P(x y)$.

To prove $\mathbf{G}(\mu)$, we first show that $\left.P \subset T\right|_{\mu+1}$. As $x, y \in V\left(\left.T\right|_{\mu+1}\right)$ and $\left.T\right|_{\mu+1}$ is a tree, $x$ and $y$ are joined by a path in $\left.T\right|_{\mu+1}$. Since $\left.T\right|_{\mu+1} \subset T$ and $T$ contains only one $x-y$ path, this path must be $P$. Notice that $S_{\mu}$ separates $y$ from every vertex $v \notin S_{\mu}$ with $\lambda(v)<\mu$ (Proposition 2.4), while $S_{\mu}$ does not separate $y$ from any $v \in \stackrel{\circ}{P}$. Therefore $\lambda(v) \geq \mu$ and hence $\lambda(v)>\mu$ for all $v \in \stackrel{\circ}{P}$. By definition of $\left.T\right|_{\mu+1}$, this implies that every $v \in \stackrel{\circ}{P}$ is contained in $\stackrel{\circ}{P}(e)$ for some $e \in E_{\mu}$. But inner vertices of different paths $P(e)$ cannot be adjacent, since $\nu(e) \neq \nu\left(e^{\prime}\right)$ and $\tau(\nu(e))=\tau\left(\nu\left(e^{\prime}\right)\right)=\mu$ for distinct $e, e^{\prime} \in E_{\mu}$. Therefore all $v \in \stackrel{\circ}{P}$ are on the same path $P(e)$, i.e. $\stackrel{\circ}{P} \subset P(e)$ for some $e \in E_{\mu}$. Since $P$ and $P(e)$ are both $B_{\mu}-B_{\mu}$ paths, this means that $P=P(e)$ and $x y=e$.

$\mathbf{G}(\mu)$ has the following useful consequence: if $P \subset T$ is a path that meets $B_{\mu}$ infinitely often but avoids $S_{\mu}$, we can turn $P$ into a path $P^{\prime} \subset T_{\mu}$ by replacing each $B_{\mu}-B_{\mu}$ path $P_{x, y} \subset P$ with the edge $x y \in E_{\mu}$. Similarly, we can contract any path $P \subset G$ onto a path $P^{\prime} \subset B_{\mu}$-recall that since $B_{\mu}$ is a convex subgraph of $G$ (Proposition 2.1), the endvertices of $P$ will be adjacent in $B_{\mu}$ :

$\mathbf{H}(\mu)$ : If $P=v_{1} v_{2} \ldots$ is a (finite or infinite) path in $G$, then $B_{\mu}$ contains a path $P^{\prime}=$ $v_{k_{1}} v_{k_{2}} \ldots$, where $V\left(P^{\prime}\right)=V(P) \cap V\left(B_{\mu}\right)$ and $k_{i}<k_{j}$ if and only if $i<j$. Moreover, if $P \subset T \backslash S_{\mu}$, then $P^{\prime} \subset T_{\mu}$.

Finally, we prove what was earlier desribed as the 'bottle-neck' property of the vertices $s_{\mu}$.

$\mathbf{I}(\mu):$ If $\mu=\tau^{k}(\nu), k \geq 0$, and $v \in G$ with $\lambda(v)=\nu$, then $s_{\mu}$ separates $v$ from $v_{0}$ in $T$ (for $\mu>0$ ).

We prove $\mathbf{I}(\mu)$ be induction on $k$. If $k=0$, then $\nu=\mu$, so $v \in T_{\mu}$. Let $P$ be the $v-v_{0}$ path in the tree $\left(\left.T_{\mu} \cup T\right|_{\mu}\right)+e_{\mu}$. By $\mathbf{B}(\mu), s_{\mu} \in P$. Replacing every edge $e \in E(P) \cap E_{\mu}$ with $P(e)$, we obtain a path in $\left.T\right|_{\mu+1}$ that joins $v$ to $v_{0}$ and contains $s_{\mu}(\operatorname{cf} . \mathbf{D}(\mu))$. Since $\left.T\right|_{\mu+1} \subset T$ and $T$ is a tree, this implies the assertion.

Suppose now that $k>0$, and that $\mathbf{I}(\mu)$ holds for all smaller values of $k$. Let $P$ be a $v-v_{0}$ path in $T$; we have to show that $s_{\mu} \in P$. By the case of $k=0, v$ is separated from $v_{0}$ by $s_{\nu}$ in $T$, so $P$ contains an $s_{\nu}-v_{0}$ path $P^{\prime}$. If $\lambda\left(s_{\nu}\right)=\tau(\nu)$, then $\mu=\tau^{k-1}\left(\lambda\left(s_{\nu}\right)\right)$, so 
$s_{\mu} \in P^{\prime}$ by the induction hypothesis. Suppose therefore that $\lambda\left(s_{\nu}\right) \neq \tau(\nu)$. Then, by $\mathbf{F}(\nu)$, $\lambda\left(s_{\nu}\right)=\tau^{2}(\nu)$ and $s_{\nu}=s_{\tau(\nu)}$. If $\tau^{2}(\nu) \geq \mu$, the assertion again follows by the induction hypothesis (as above). But if $\tau^{2}(\nu)<\mu$, then $k=1$ and $\mu=\tau(\nu)$, giving $s_{\nu}=s_{\tau(\nu)}=s_{\mu}$. This completes the proof of $\mathbf{I}(\mu)$.

\section{4. $T$ is end-complete}

It will be convenient in this and the next section to call a ray $P \subset G$ centred on $B_{\mu}$ if $P$ meets $B_{\mu}$ infinitely often, and uncentred if $V(P) \cap V\left(B_{\mu}\right)$ is finite for every $\mu<\sigma$.

Let $P$ be a given ray in $G$; we have to find a ray $Q \subset T$ such that $P \sim Q$.

We first suppose that $P$ is centred on some $B_{\mu}, \mu<\sigma$. Since any ray ${ }_{Q} Q$ is equivalent to $P$ as soon as it is equivalent to some tail of $P$, we may assume that $P \cap S_{\mu}=\emptyset$. Let $P^{\prime} \subset B_{\mu}$ be the path obtained from $P$ by $\mathbf{H}(\mu)$; then $P^{\prime}$ is a ray in $B_{\mu} \backslash S_{\mu}$. As $T_{\mu}$ is an end-faithful spanning tree of $B_{\mu} \backslash S_{\mu}$, it contains a ray $Q^{\prime}$ with $P^{\prime} \underset{B_{\mu} \backslash S_{\mu}}{\sim} Q^{\prime}$, and hence $P^{\prime} \underset{G^{\prime}}{\sim} Q^{\prime}$. Let $Q$ be obtained from $Q^{\prime}$ by replacing each edge $e \in E\left(Q^{\prime}\right) \cap E_{\mu}$ with the path $P(e)$. Then $\left.Q \subset T\right|_{\mu+1} \subset T$, and $Q$ is a ray (by $\mathbf{C}(\mu)$ ). As $P^{\prime}{\widetilde{G^{\prime}}}^{Q^{\prime}}$, clearly $P \underset{\sigma^{\prime}}{\sim} Q$. By Lemma 3.1, this implies that $P \underset{G}{\sim} Q$.

Let us from now on suppose that $P$ is uncentred. Recall that the factors $B_{\lambda}$ in our decomposition $F$ of $G^{\prime}$ are the vertices of the decomposition tree $T_{F}$. For given rays $P \subset G$ and $R=B_{\lambda_{0}} B_{\lambda_{1}} \ldots \subset T_{F}$, let us set

$$
I(P, R):=\left\{i \in \mathbb{N} \mid \lambda_{i} \in \Lambda(P)\right\} .
$$

The following two lemmas relate the uncentred rays in $G$ to rays in $T_{F}$.

Lemma 4.1. For every uncentred ray $P \subset G$, there exists a ray $R$ from $B_{0}$ in $T_{F}$ such that $I(P, R)$ is infinite.

Proof. Let $T(P)$ be the union of all $B_{\lambda}-B_{0}$ paths in $T_{F}$ with $\lambda \in \Lambda(P)$. $T(P)$ is a subtree of $T_{F}$. For vertices $B^{\prime}, B^{\prime \prime}$ of $T_{F}$, let us say that $B^{\prime}$ precedes $B^{\prime \prime}$ if $B^{\prime}<B^{\prime \prime}$ but there is no vertex $B \in T_{F}$ of the form $B=B_{\lambda}, \lambda \in \Lambda(P)$, such that $B^{\prime}<B<B^{\prime \prime}$. Let us prove the following:

Each vertex of $T(P)$ precedes at most finitely many vertices $B_{\lambda} \in T(P)$ with $\lambda \in \Lambda(P)$.

Suppose (3) fails, and let $B$ be a vertex of $T(P)$ that precedes every vertex in some infinite set $U \subset\left\{B_{\lambda} \mid \lambda \in \Lambda(P)\right\}$. We show that whenever $B^{\prime}, B^{\prime \prime}$ are distinct elements of $U$, any subpath $P^{\prime}=v \ldots w$ of $P$ with $v \in B^{\prime} \backslash B$ and $w \in B^{\prime \prime} \backslash B$ passes through $B$. Since $P$ has a vertex in $B_{\lambda} \backslash B$ for every $B_{\lambda} \in U$ (by $B_{\lambda}>B$ and the definition of $U$ ), this means that $P$ meets $B$ infinitely often, contrary to our assumption that $P$ is uncentred. 
Let $B^{\prime}, B^{\prime \prime}$ and $P^{\prime}$ be given as stated. $B^{\prime}$ and $B^{\prime \prime}$ are incomparable in $T_{F}$, because $B$ precedes both of them. Let $\widetilde{B}$ be the maximal vertex of $T_{F}$ that satisfies $\widetilde{B}<B^{\prime}$ as well as $\widetilde{B}<B^{\prime \prime}$. Then $\widetilde{B} \geq B$. Moreover, $\widetilde{B}$ lies on the $B^{\prime}-B^{\prime \prime}$ path in $T_{F}$, so $P^{\prime} \cap \widetilde{B} \neq \emptyset$ by Proposition 2.3. Let $u \in P^{\prime} \cap \widetilde{B}$. Clearly $B_{\lambda(u)} \leq \widetilde{B}$, so $B_{\lambda(u)}$ and $B$ are comparable, because also $B \leq \widetilde{B}$. Since $B_{\lambda(u)} \leq \widetilde{B}<B^{\prime}$ and $B_{\lambda(u)} \leq \widetilde{B}<B^{\prime \prime}$ but $B$ precedes $B^{\prime}$ and $B^{\prime \prime}$, we have $B \nless B_{\lambda(u)}$, and therefore $B \geq B_{\lambda(u)}$ (see the first part of Figure 2). Thus $B$ lies on the $B^{\prime}-B_{\lambda(u)}$ path in $T_{F}$, which implies that $P_{u, v} \cap B \neq \emptyset$ (by Proposition 2.3). This completes the proof of (3).
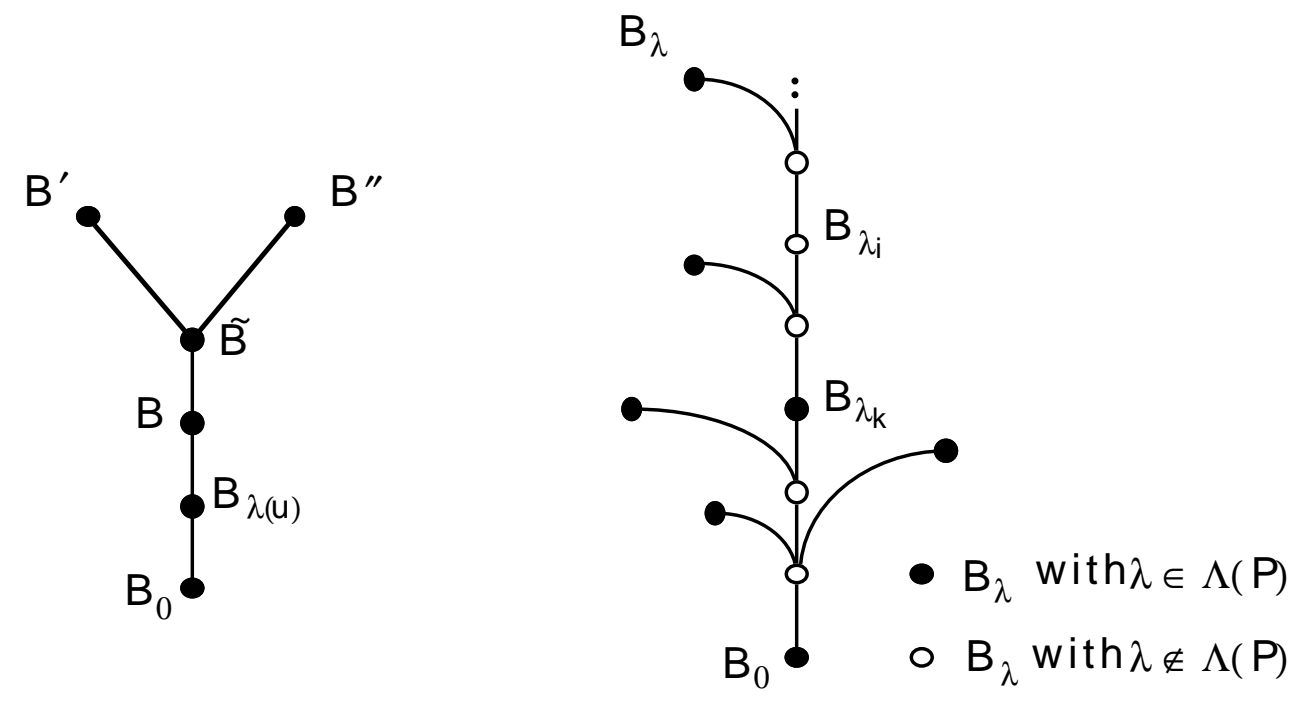

Figure 2. Vertices of $T(P)$ in the proof of (3)

As a first consequence of (3), we note that $T(P)$ is locally finite: if $B \in T(P)$ has infinitely many neighbours $B^{\prime}>B$, and $B_{\lambda}^{\prime}$ is the smallest vertex of $T(P)$ with $B^{\prime} \leq B_{\lambda}^{\prime}$ and $\lambda \in \Lambda(P)$ (recall the construction of $T(P)$ ), then $B$ precedes $B_{\lambda}^{\prime}$, and all these $B_{\lambda}^{\prime}$ 's are distinct. By König's theorem, $T(P)$ therefore contains a ray $R=: R(P)$, say $R=$ $B_{\lambda_{0}} B_{\lambda_{1}} \ldots$. Since $B_{0} \in T(P)$, we may assume that $\lambda_{0}=0$.

It remains to show that infinitely many of the indices $\lambda_{i}$ are in $\Lambda(P)$. Suppose not, and let $k$ be maximal with $\lambda_{k} \in \Lambda(P)$. Then no $B_{\lambda_{i}}$ with $i>k$ is of the form $B_{\lambda}, \lambda \in \Lambda(P)$, but, by construction of $T(P)$, each of them precedes such a $B_{\lambda}$, and is in turn preceded by $B_{\lambda_{k}}$ (see the second part of Figure 2). Since for every $B_{\lambda} \in T_{F}$ there are only finitely many vertices $B \in T_{F}$ with $B_{\lambda}>B$ but $\left\{B_{\lambda_{i}} \mid i>k\right\}$ is infinite, this means that $B_{\lambda_{k}}$ precedes infinitely many vertices $B_{\lambda}$ with $\lambda \in \Lambda(P)$, contrary to (3).

Lemma 4.2. If $P$ is an uncentred ray in $G$ and $R=B_{\lambda_{0}} B_{\lambda_{1}} \ldots$ is a ray from $B_{0}$ in $T_{F}$ such that $I=I(P, R)$ is infinite, then every tail of $P$ meets every $S_{\lambda_{n}}$ with sufficiently large $n \in \mathbb{N}$. 
Proof. Let $P^{\prime}$ be a given tail of $P$. Pick $k \in I^{\prime}$, where

$$
I^{\prime}:=I \backslash\left\{i \in I \mid \lambda_{i} \in \Lambda\left(P \backslash P^{\prime}\right)\right\}
$$

Since $P \backslash P^{\prime}$ is finite but $I$ is infinite, $I^{\prime}$ is also infinite, and $\lambda_{i} \in \Lambda\left(P^{\prime}\right)$ for every $i \in I^{\prime}$. We show that $P^{\prime}$ meets every $S_{\lambda_{n}}$ with $k<n \in \mathbb{N}$. Let such $n$ be given, and let $i \geq n$ with $i \in I^{\prime}$. Let $u, v \in P^{\prime}$ with $\lambda(u)=\lambda_{k}$ and $\lambda(v)=\lambda_{i}$. Since $\lambda_{k}<\lambda_{n}$, we have $\left.u \in G\right|_{\lambda_{n}}$, so $v$ and $u$ are separated by $S_{\lambda_{n}}$ in $G^{\prime}$ unless $u$ is itself in $S_{\lambda_{n}}$ (by Proposition 2.4). Hence $P_{u, v}^{\prime} \cap S_{\lambda_{n}} \neq \emptyset$ as claimed.

Equipped with Lemmas 4.1 and 4.2, we can now tackle the second case in our proof of the end-completeness of $T$ in $G$. Let $P$ be a given uncentred ray in $G$, and let $R=$ $B_{\lambda_{0}} B_{\lambda_{1}} \ldots$ be as provided by Lemma 4.1. Define $Q:=\bigcup_{n=0}^{\infty} Q_{n}$, where $Q_{n}$ is the $s_{\lambda_{n}-}$ $s_{\lambda_{n+1}}$ path in $T$ (and $s_{\lambda_{0}}:=v_{0}$ ). We shall first prove that $Q$ is a ray, and then show that $Q \underset{G}{\sim} P$.

In order to prove that $Q$ is a path, we show that for different $n \geq 0$ the vertices $v \in Q_{n}-s_{\lambda_{n}}$ have distinct $\lambda(v)$, so the paths $Q_{n}-s_{\lambda_{n}}$ must be disjoint for different $n$. For every $\lambda<\sigma$, let $\lambda^{\prime}$ be such that $B_{\lambda^{\prime}}$ is the maximal vertex on $R$ with $B_{\lambda^{\prime}} \leq B_{\lambda}$. (To see that $\lambda^{\prime}$ exists, recall that $R$ is a ray from $B_{0}$, which is the root of $T_{F}$.) We prove the following:

$$
v \in Q_{n}-s_{\lambda_{n}}, n \geq 0 \quad \Rightarrow \quad \lambda^{\prime}(v)=\lambda_{n}
$$

We apply induction on $n$. Let $n \geq 0$, and suppose the assertion holds for all $i<n$. Then $\bigcup_{i=0}^{n-1} Q_{i}$ is (empty or) the $v_{0}-s_{\lambda_{n}}$ path in $T$. By $\mathbf{F}\left(\lambda_{n+1}\right)$, we have either $\lambda\left(s_{\lambda_{n+1}}\right)=$ $\lambda_{n}$ or $s_{\lambda_{n+1}}=s_{\lambda_{n}}$; recall that $\tau\left(\lambda_{n+1}\right)=\lambda_{n}$. In the latter case $Q_{n}-s_{\lambda_{n}}=\emptyset$, so there is nothing to prove; we shall therefore assume that $\lambda\left(s_{\lambda_{n+1}}\right)=\lambda_{n}$. Then $s_{\lambda_{n+1}} \in B_{\lambda_{n}} \backslash S_{\lambda_{n}}$. Recall that $e_{\lambda_{n}}$ is an edge of $G^{\prime}$ joining $s_{\lambda_{n}}$ to a vertex of $B_{\lambda_{n}} \backslash S_{\lambda_{n}}$, say to $x$. Let $Q_{n}^{\prime}$ be the $s_{\lambda_{n}}-s_{\lambda_{n+1}}$ path in $B_{\lambda_{n}}$ consisting of $e_{\lambda_{n}}$ followed by the $x-s_{\lambda_{n+1}}$ path in $T_{\lambda_{n}}$. (Recall that $T_{\lambda_{n}}$ is a spanning tree of $B_{\lambda_{n}} \backslash S_{\lambda_{n}}$.) If we replace every edge $e \in E\left(Q_{n}^{\prime}\right) \cap E_{\lambda_{n}}$ with the path $P(e) \subset T$, we obtain an $s_{\lambda_{n}}-s_{\lambda_{n+1}}$ path in $T$ (cf. $\mathbf{C}\left(\lambda_{n}\right)$ ). Since $T$ is a tree, this is the unique $s_{\lambda_{n}}-s_{\lambda_{n+1}}$ path in $T$, and therefore equal to $Q_{n}$.

To complete the proof of (4), it remains to show that $\lambda^{\prime}(v)=\lambda_{n}$ for every $v \in \stackrel{P}{P}(e)$ with $e \in E\left(Q_{n}^{\prime}\right) \cap E_{\lambda_{n}}$. By definition of $P(e)$, we have $\lambda(v)=\nu(e)$ for $v \in \stackrel{\circ}{P}(e)$ and $\tau(\nu(e))=\lambda_{n}$. Therefore $\lambda^{\prime}(v)=\lambda_{n}$ unless $\lambda^{\prime}(v)=\lambda(v)=\lambda_{n+1}$.

Before we show that $\lambda^{\prime}(v)$ must be $\lambda_{n}$ rather than $\lambda_{n+1}$, let us note that certainly $\lambda^{\prime}(v)>\lambda_{i}$ for all $i<n$, and therefore $v \notin Q_{0} \cup \ldots \cup Q_{n-1}$ by the induction hypothesis. Thus $\stackrel{\circ}{Q}_{n} \cap \bigcup_{i=0}^{n-1} Q_{i}=\emptyset$, and $Q_{0} \cup \ldots \cup Q_{n}$ is the $v_{0}-s_{\lambda_{n+1}}$ path in $T$.

Let us now resume our proof that $\lambda^{\prime}(v)=\lambda_{n}$ for any given $v \in \stackrel{\circ}{P}(e)$ and $e \in E\left(Q_{n}^{\prime}\right) \cap$ $E_{\lambda_{n}}$. As shown above, all we have to check is that $\lambda(v) \neq \lambda_{n+1}$. This, however, follows from the definition of $e_{\lambda_{n+1}}$ and $s_{\lambda_{n+1}}$ : if $\lambda(v)=\lambda_{n+1}$, then $\lambda_{n+1}$ is of type 2 (because $\left.\left.\stackrel{\circ}{P} \subset T\right|_{\lambda_{n+1}} \cap\left(B_{\lambda_{n+1}} \backslash S_{\lambda_{n+1}}\right)\right)$ and $s_{\lambda_{n+1}}$ lies on the $v-v_{0}$ path in $\left.T\right|_{\lambda_{n+1}} \subset T$-which 
contradicts the fact that $v \in \stackrel{Q}{Q}_{n}$ and $Q_{0} \cup \ldots \cup Q_{n}$ is the $v_{0}-s_{\lambda_{n+1}}$ path in $T$. This completes the proof of (4), showing that $Q$ is a path.

The proof that $Q$ is infinite, and therefore a ray, is now straightforward. As $V(Q) \supset$ $\left\{s_{\lambda_{n}} \mid n \in \mathbb{N}\right\}, Q$ can only be finite if infinitely many $s_{\lambda_{n}}$ 's coincide. This however is ruled out by $(2)$.

We have shown that $Q$ is a ray in $T$ that passes through every vertex $s_{\lambda_{i}}, i \in \mathbb{N}$. In order to prove that $Q$ is equivalent to $P$ in $G$, let $U$ be a given finite set of vertices of $G$, and let $P^{\prime}$ and $Q^{\prime}$ be the tails of $P$ and $Q$ in $G-U$, respectively. We have to show that $G-U$ contains a $P^{\prime}-Q^{\prime}$ path. By Lemma 4.2 and the definition of $Q$, we can find an $n \in \mathbb{N}$ such that $P^{\prime} \cap S_{\lambda_{n}} \neq \emptyset$, say $s \in P^{\prime} \cap S_{\lambda_{n}}$, and $s_{\lambda_{n}} \in Q^{\prime}$. If $s=s_{\lambda_{n}}$ or $s s_{\lambda_{n}} \in E(G)$, we are done. But otherwise $s s_{\lambda_{n}} \in E\left(G^{\prime}\right) \backslash E(G)$, so $G$ contains uncountably many independent $s^{-} s_{\lambda_{n}}$ paths, one of which avoids $U$.

\section{5. $T$ is end-respecting}

Lemma 5.1. Suppose $P_{1}, P_{2}$ are rays in $G, P_{1}$ is centred on $B_{\mu}(\mu<\sigma)$, and $P_{1} \underset{G}{\sim} P_{2}$. Then $P_{2}$ is also centred on $B_{\mu}$.

Proof. If $P_{2}$ is centred at all, say on $\nu$, then clearly $\nu=\mu$ : if $\nu \neq \mu$, without loss of generality $\nu>\mu$, then $S_{\nu}$ separates infinitely many vertices of $P_{1}$ from infinitely many vertices of $P_{2}$ in $G^{\prime}$ (Proposition 2.4), which contradicts our assumption that $P_{1} \widetilde{G} P_{2}$.

Suppose therefore that $P_{2}$ is not centred. Let $R=B_{\lambda_{0}} B_{\lambda_{1}} \ldots$ be a ray in $T_{F}$ such that $I\left(P_{2}, R\right)$ is infinite (Lemma 4.1). For every $i \in I\left(P_{2}, R\right)$, let $v_{i}$ be a vertex on $P_{2}$ with $\lambda\left(v_{i}\right)=\lambda_{i}$. As at most finitely many $B_{\lambda_{n}}$ can be such that $B_{\mu} \geq B_{\lambda_{n}}$, there exists $k \in \mathbb{N}$ with $B_{\mu} \geq B_{\lambda_{k}}$. By Proposition 2.4, $S_{\lambda_{k}}$ separates every $v_{i}$ with $i \geq k$ from every vertex $u \in B_{\mu} \backslash S_{\lambda_{k}}$. Thus the finite set $V\left(S_{\lambda_{k}}\right)$ separates infinitely many vertices of $P_{1}$ from infinitely many vertices of $P_{2}$ in $G^{\prime}$, again contradicting $P_{1} \underset{G}{\sim} P_{2}$.

For our proof that $T$ respects the ends of $G$, let rays $P_{1}, P_{2} \subset T$ with $P_{1} \underset{G}{\sim} P_{2}$ be given. We have to show that $P_{1} \underset{T}{\sim} P_{2}$. We shall distinguish two cases: that $P_{1}$ and $P_{2}$ are both centred on the same $B_{\mu}$, and that $P_{1}$ and $P_{2}$ are both uncentred. By Lemma 5.1 this distinction is exhaustive.

Case 1: $P_{1}$ and $P_{2}$ are centred on $B_{\mu}, \mu<\sigma$.

Since $S_{\mu}$ is finite, we may assume without loss of generality that $P_{i} \cap S_{\mu}=\emptyset, i=1,2$. Let us choose an infinite sequence $Q_{1}, Q_{2}, \ldots$ of disjoint paths in $G$ as follows. Having definied $Q_{1}, \ldots, Q_{n-1}$ for some $n \in \mathbb{N}$, consider the tails $P_{1}^{n}$ and $P_{2}^{n}$ of $P_{1}$ and $P_{2}$ in $G \backslash \bigcup_{i=1}^{n-1} Q_{i}$. If $P_{1}^{n} \cap P_{2}^{n} \neq \emptyset$, set $Q_{n}:=\left\{q_{n}\right\}$ for some vertex $q_{n} \in P_{1}^{n} \cap P_{2}^{n}$. If $P_{1}^{n} \cap P_{2}^{n}=\emptyset$, let 
$Q_{n}=q_{n}^{1} \ldots q_{n}^{2}$ be a path in $G \backslash\left(S_{\mu} \cup \bigcup_{i=1}^{n-1} Q_{i}\right)$ with $q_{n}^{i} \in P_{i} \cap B_{\mu}, \quad i=1,2$. Note that $Q_{n}$ exists, because by assumption $P_{1}$ and $P_{2}$ are equivalent in $G$ and centred on $B_{\mu}$.

Let $P_{1}^{\prime}$ and $P_{2}^{\prime}$ correspond to $P_{1}$ and $P_{2}$ as in $\mathbf{H}(\mu)$, and let $Q_{n}^{\prime}$ correspond to $Q_{n}$, $n \in \mathbb{N}$. Then $Q_{1}^{\prime}, Q_{2}^{\prime}, \ldots$ is an infinite sequence of disjoint paths in $B_{\mu} \backslash S_{\mu}$, each joining a vertex of $P_{1}^{\prime}$ to one of $P_{2}^{\prime}$. Therefore $P_{1}^{\prime} \underset{B_{\mu} \backslash S_{\mu}}{\sim} P_{2}^{\prime}$. As $P_{i} \subset T \backslash S_{\mu}$ by assumption, $\mathbf{H}(\mu)$ implies that $P_{i}^{\prime} \subset T_{\mu}, \quad i=1,2$. But $T_{\mu}$ is an end-respecting spanning tree of $B_{\mu} \backslash S_{\mu}$, so $P_{1}^{\prime} \underset{T_{\mu}}{\sim} P_{2}^{\prime}$. As $T_{\mu}$ is a tree, this equivalence means that $V\left(P_{1}^{\prime}\right) \cap V\left(P_{2}^{\prime}\right)$ is infinite. Therefore $V\left(P_{1}\right) \cap V\left(P_{2}\right)$ is infinite, too (recall that $V\left(P_{i}^{\prime}\right) \subset V\left(P_{i}\right), i=1,2$ ), so $P_{1} \underset{T}{\sim} P_{2}$ as claimed.

Case 2: $P_{1}$ and $P_{2}$ are both uncentred.

We shall assume, without loss of generality, that $P_{1}$ and $P_{2}$ are rays from $v_{0}$. For $i=1,2$, let $R_{i}$ be a ray from $B_{0}$ in $T_{F}$ such that $I_{i}:=I\left(P_{i}, R_{i}\right)$ is infinite (by Lemma 4.1).

Let us use the equivalence of $P_{1}$ and $P_{2}$ in $G$ to show that $R_{1}=R_{2}$. Suppose $R_{1} \neq R_{2}$, and let $B_{\mu}$ be the first (= minimal) vertex of $R_{1}$ that is not on $R_{2}$. Then $B \nsupseteq B_{\mu}$ for every $B \in V\left(R_{2}\right)$, because $R_{2}$ is a ray from $B_{0}$. By Proposition 2.4 , therefore, and the fact that $I_{1}$ and $I_{2}$ are infinite, $S_{\mu}$ separates infinitely many vertices of $P_{1}$ from infinitely many vertices of $P_{2}$ in $G^{\prime}$. As $S_{\mu}$ is finite, this contradicts our assumption that $P_{1} \underset{G}{\sim} P_{2}$.

Thus $R_{1}=R_{2}=: R$, say $R=B_{\lambda_{0}} B_{\lambda_{1}} \ldots$ As $I_{1}$ and $I_{2}$ are infinite, $\mathbf{I}\left(\lambda_{n}\right)$ implies that $P_{1}$ and $P_{2}$ contain $s_{\lambda_{n}}$, for every $n \in \mathbb{N}$. (Recall that $P_{1}$ and $P_{2}$ are rays from $v_{0}$.) By (2) therefore, $P_{1}$ and $P_{2}$ have infinitely many vertices in common, giving $P_{1} \underset{T}{\widetilde{T}} P_{2}$ as desired.

\section{References}

[1] B. Bollobás, Graph Theory, An Introductory Course, Springer-Verlag, New York 1979.

[2] R. Diestel, Graph Decompositions - a study in infinite graph theory, Oxford University Press, Oxford 1990.

[3] R. Diestel, The structure of TK$K_{a}$-free graphs, submitted.

[4] R. Diestel (Ed.), Directions in Infinite Graph Theory, Annals of Discrete Mathematics, in preparation.

[5] R. Halin, Über unendliche Wege in Graphen, Math. Ann. 157 (1964), 125-137.

[6] R. Halin, Automorphisms and endomorphisms of infinite locally finite graphs, Abh. Math. Sem. Univ. Hamburg 39 (1973), 251-283.

[7] R. Halin, Simplicial decompositions of infinite graphs, in: (B. Bollobás, Ed.) Advances in Graph Theory (Annals of Discrete Mathematics 3), North-Holland Publ. Co., Amsterdam/London 1978.

[8] H. A. Jung, Wurzelbäume und unendliche Wege in Graphen, Math. Nachr. 41 (1969), 1-22.

[9] D. König, Theorie der endlichen und unendlichen Graphen, Leipzig 1936.

[10] N. Polat, Topological aspects of infinite graphs, in: (G. Hahn et al., Eds.) Cycles and Rays, NATO ASI Ser. C, Kluwer Academic Publishers, Dordrecht 1990.

[11] N. Polat, Spanning trees of infinite graphs, preprint 1989.

[12] N. Seifter, The action of nilpotent groups on infinite graphs, Mh. Math. 99 (1985), 323-333. 
[13] P. D. Seymour and R. Thomas, An end-faithful counterexample, preprint 1989.

[14] M. E. Watkins, Infinite paths that contain only shortest paths, J. Combin. Theory B 41 (1986), 341355.

Reinhard Diestel, SFB 343, Bielefeld University 